\title{
A Novel Algorithm to Design an Efficient siRNA by Combining the Pre Proposed Rules of siRNA Designing
}

\author{
Shishir Kumar Gupta ${ }^{1 *}$, Bashir Akhlaq Akhoon², Mugdha Srivastava', Shailendra Kumar Gupta ${ }^{3}$ \\ ${ }^{1}$ Society for Biological Research \& Rural Development, Lucknow, 226010, UP, India \\ ${ }^{2}$ Centre of Bioinformatics, Department of Biotechnology, SMVD University, Jammu, India \\ ${ }^{3}$ Indian Institute of Toxicology Research, Lucknow, CSIR, India
}

\begin{abstract}
Short interfering RNAs (siRNAs) can be used to suppress gene expression and have a lot of potential applications in therapy, yet how to design an effective siRNA is still under consideration. Numerous siRNA design tools have been developed recently. The set of candidates reported by these tools is usually large and often contains ineffective siRNAs. We initiated with the filtering of ineffective siRNAs, specifically, the current work establishes relationship between mentioned theoretically calculated property of subsequences and their efficiency for RNAi for any input set of experimental data. We combined some reported algorithms and designed a new algorithm that mainly focuses on stability and off-targeting to design siRNA sequences.
\end{abstract}

Keywords: siRNA; Algorithm; Rules; siRNA designing

\section{Introduction}

RNA Interference (RNAi) is powerful specific process which is actively carried out by special mechanisms in the cell. RNA interference (RNAi) is a gene regulatory pathway triggered in response to double-stranded RNA (dsRNA) (Hannon, 2002). Double-stranded (ds) RNA induces the sequence-specific posttranscriptional gene silencing of cognate genes in numerous organisms (Cogoni and Macino, 2000; Hutvagner and Zamore, 2002a; Denli and Hannon, 2003). The multidomain ribonuclease III enzyme Dicer excises long dsRNA into duplexes of 21-23 nucleotides (nt) termed short interfering RNAs (Bernstein et al., 2001; Ketting et al., 2001; Knight and Bass, 2001), which direct the cleavage of complementary mRNA targets, a process known as RNA interference (Fire et al., 1998). Prior to target mRNA recognition, an siRNA duplex goes through an ATP-dependent unwinding process and one strand over the other is often preferentially loaded onto the RNA-induced silencing complex (RISC), the multiple turnover enzyme complex that mediates endonucleolytic cleavage in the RNAi pathway. The RISC is guided to cleave target mRNAs sharing perfect complementarity across the center of the complementary siRNA strand in the absence of high-energy cofactors (Hammond et al., 2001; Nykanen et al., 2001; Hutvagner and Zamore, 2002; Martinez et al., 2002). siRNAs are not the only products of Dicer. Natural dsRNA-encoding genes, named microRNA (miRNA) genes, encode RNA products of $70 \mathrm{nt}$ that are predicted to form imperfect hairpin structures and are processed by Dicer to mature 21-23-nt miRNAs (Grishok et al., 2001; Hutvagner et al., 2001).

Gene silencing mediated by siRNA is not transparent at the mRNA level, but apparently detectable at the protein level. Elimination of the off-target silencing mediated by the 5 'seed pairing is extremely challenging, and most siRNAs will have a number of unintended targets affected by 5 'seed pairing. More than one siRNA to a given target is commonly used in discovery research to ensure a single phenotype resulting from on-target gene silencing by most siRNAs used. "Multiple siRNAs per target" approach is acceptable for discovery research but fails in potential therapeutic applications (Jayasena, 2006). The further refinement of design algorithms is necessary for siRNAs especially aimed for therapeutic developments. It is expected to merge miRNA target prediction algorithms with those developed for siRNA design to eliminate candidate siRNAs with potential off target gene silencing through the undesirable miRNA-related mechanism (Jayasena, 2006).

While it is desirable to incorporate all of the selection rules into a computer aided siRNA designing algorithm, the complication at the moment is how to rank those published rules, especially when some of the rules are contradictive. Currently, many computer aided siRNA design tools have been published and some of those have been made accessible through websites. However, none of those tools has successfully incorporated all the rules, and most of them treat their employed rules without much differentiation. In general, the existing tools adopt a set of rules and assign each rule an equal or different score, and each siRNA sequence is scored against every rule and only those sequences scoring above a predefined point are selected as valid siRNA sequences. Such a simple selection procedure does not accommodate the possibility that some rules are critical for the validity of a siRNA sequence (must be met), while some rules can only affect the efficiency of the siRNA sequence (Hong et al., 2006). Meanwhile, those web-based tools only provide users very limited flexibility, and users cannot reorganize the selection rules based on their own preferences or recent research data (Hong et al., 2006). Although the actual mechanism of which is still unclear, the off-target effect of siRNA is largely attributed to partial sequence homology between siRNA and its unintended targets. Table 1 listed comparative study of about all computational tools available for siRNA designing on the basis of various design rules and various siRNA design parameters.

*Corresponding author: Shishir K Gupta, M.Sc., Society for Biological Research \& Rural Development, The Bioinformatica Solutions, 1/8, Vijay Khand, Gomti Nagar, 226010, Lucknow, UP, India, Tel: +91- 9451157798, +91 522 2391518; E-mail: shishir.bioinfo@gmail.com

Received January 13, 2010; Accepted February 08, 2010; Published February 08,2010

Citation: Gupta SK, Akhoon BA, Srivastava M, Gupta SK (2010) A Novel Algorithm to Design an Efficient siRNA by Combining the Pre Proposed Rules of siRNA Designing. J Comput Sci Syst Biol 3: 005-009. doi:10.4172/jcsb. 1000048

Copyright: () 2010 Gupta SK, et al. This is an open-access article distributed under the terms of the Creative Commons Attribution License, which permits unrestricted use, distribution, and reproduction in any medium, provided the original author and source are credited. 
Citation: Gupta SK, Akhoon BA, Srivastava M, Gupta SK (2010) A Novel Algorithm to Design an Efficient siRNA by Combining the Pre Proposed Rules of siRNA Designing. J Comput Sci Syst Biol 3: 005-009. doi:10.4172/jcsb.1000048

\begin{tabular}{|c|c|c|}
\hline Tool/Server & Rules & Comments \\
\hline Ambion & Tuschl & No rank/score; link to NCBI Blast \\
\hline Dharmacon & Reynolds & $\begin{array}{l}\text { Score/Rank; Automatically runs blast } \\
\text { Can't see results or conditions of Blast }\end{array}$ \\
\hline Deqor & Own set of Rules & $\begin{array}{l}\text { Built for siRNA and esiRNA; Score/Rank; Automatically runs Blast, } \\
\text { can control conditions }\end{array}$ \\
\hline EMBOSS & Tuschl & Score/Rank; No Blast \\
\hline GenScript & Combination of Tuschl, Reynolds, Ui-Tei & $\begin{array}{l}\text { Score/Rank; Blast/Smith Waterman vs. proprietary database of EST + } \\
\text { mRNA }\end{array}$ \\
\hline Interagon & Allows use of 8 different algorithms & $\begin{array}{l}\text { Requires registration; Blast performed automatically against Mouse } \\
\text { Ensemble transcripts only; }\end{array}$ \\
\hline OptiRNAi & Tuschl & Score/Rank; Link to Genome Blast \\
\hline Promega & Combination of Rules & Link to NCBI Blast, no control over parameters \\
\hline Qiagen & Tuschl Rules + extra parameters & Score/Rank; Link to NCBI Blast, no control of parameters \\
\hline SFold & Combination of folding, Reynolds, Tuschl & Score, no rank; No Blast \\
\hline SiDE & Combination of different rules & $\begin{array}{l}\text { Built for high throughput (No option to add sequence, just accession } \\
\text { numbers); checks blast against Ensemble transcripts }\end{array}$ \\
\hline siDirect & Ui-Tei, option for custom rules & $\begin{array}{l}\text { No Score/Rank; Automatic Blast, private database, no control over } \\
\text { parameters, but can see results }\end{array}$ \\
\hline $\begin{array}{l}\text { siRNA } \\
\text { wizard }\end{array}$ & Mixed Rules & $\begin{array}{l}\text { Gives top 10, with "recommended" in blue; automatic blast against } \\
\text { local mRNA database }\end{array}$ \\
\hline siSearch & $\begin{array}{l}\text { Stockholm Rules, also Ui-Tei, Amarzguioui and } \\
\text { Prydz, Reynolds allows custom rules }\end{array}$ & various output formats; Blast with choices; \\
\hline SIR & Mixed rules, scoreing based & Based on Weighted scoreing system, with siRNA database. \\
\hline
\end{tabular}

\section{Materials and Methods}

Tools used

(a) RNA structure 4.5: The RNA structure 4.5 package was used which consist of programs for the prediction and comparison of RNA secondary structures as well as siRNA energy calculation.

(b) Java 1.5.0.7: Programming language java used for source code design purpose, because it is robust platform independent object oriented language which very well fitted to our corresponding objective.

(c) MySQL: MySQL 5.0 is a relational database which was used for data storage purpose.

(d) Jakarta Tomcat 5.0: The web container Jakarta Tomcat was used as the presentation layer which is an implementation of the new Servlet 2.4 and JSP 2.0 API specifications.

(e) JSP/SERVLET: JSP files are mixed in with static HTML pages, images, and other resources in the public section. Moreover the replacement of Java code scriptlets with JSP tags was performed to improve the readability of the tool.

(f) JDBC: JSP/java application needs to communicate with a database then JDBC connector was used.

\section{Hardware and environment}

Compaq Intel 2.80 PC with a Pentium dual core processor running on the Microsoft Windows server 2003 operating system was used for development. Partial work was performed in Linux environment using Linux SUSE 10.1 operating system.

\section{Other used software and packages}

MATLAB 6.5 statistical software was used to analyze the siRNA data and various online siRNA design and validation tools were used.

\section{Data/database used}

(a)Sequence data: NCBI RefSeq database (http:// J Comput Sci Syst Biol www.ncbi.nlm.nih.gov/refseq) was used as the experiment mRNA dataset. In order to test the efficacy of designed algorithm, Homo sapiens Alzheimer's mRNA sequence (Accession no. NM_001007532.2) were used as reference sequence. Comparative analysis of siRNA prediction and their effectiveness were tested for Ambion tool, siRNA program of mEMBOSS 6.0.1 and our tool based on designed algorithm (Figure 1) using Oligowalk tool of RNA structure 4.5 package.

(b) siRNA database: Ambion siRNA database and SIR were used for comparison between design and pre-existing siRNA sequences.

(c) BLAST and Smith-Waterman Search: It has been suggested that the un-detected sequence homology by BLAST search play major role in designing siRNA. In present work, we employed two filters to screen for the possible off-target effect. First, BLAST was applied to identify and remove any off-target matches for all the siRNA sequences that survive the three-phase selection procedure. Then, the remaining sequences are screened by the Smith-Waterman search.

Rules aplied for pediction and dsigning of siRNAs

(a) Amarzguioui Rules:

- Duplex End A/U differential > 0 .

- Strong binding of 5'sense strand.

- No U at position 1.

- Presence of A at position 6.

- Weak binding of 3'sense strand.

- No $\mathrm{G}$ at position 19.

(b) Tuschl Rules: It is an early but still used attempt to design working siRNA duplexes leads to the following empirical rules:

- Select targeted region from a given cDNA (compatible DNA created as corresponding to mRNA) sequence beginning 50-100nt downstream of start codon.

- First search for 23-nt sequence motif AA(N19)TT Volume 3(1): 005-009 (2010) - 006 


\section{Journal of Computer Science \& Systems Biology - Open Access}

\section{JCSB/Vol.3 Issue 1}

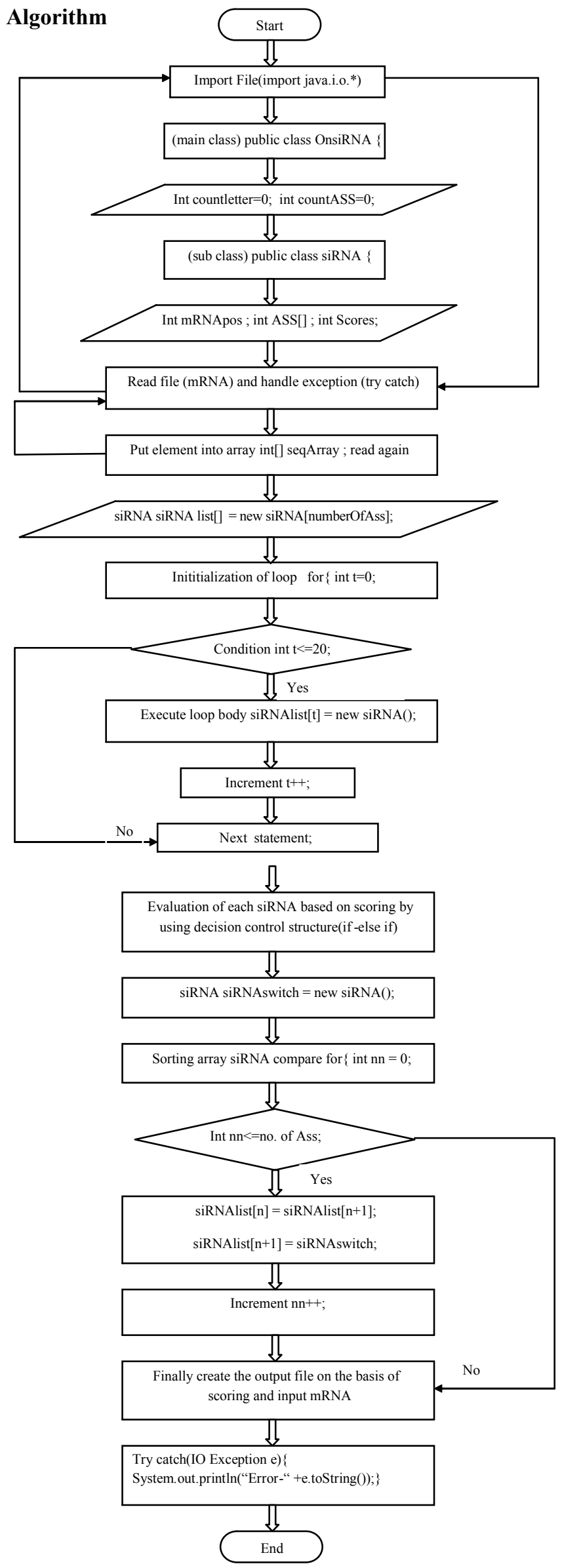

- After it, search for 23-nt sequence motif NA(N21) and con vert the 3' end of the sense siRNA to TT

- Finally search for NAR(N17)YNN, where $R=\{A, G\}$ and $\mathrm{Y}=\{\mathrm{C}, \mathrm{T}\}$

- Target sequence should have a GC content of around 50\%.

(c) Hohjoh Rules: These rules represents newly designed siRNA duplexes as, "fork- siRNA duplexes", that can enhance RNAi activity over conventional siRNA duplexes in cultured mammalian cells.

- "Fork-siRNA" mismatch at the 3' sense strand-siRNA

- $\mathrm{G} / \mathrm{C}$ at position 1 (sense strand)

- $\mathrm{A} / \mathrm{U}$ at position 19 (sense strand)

- A/U at position 8 (sense strand)

(d) Ui-Tei Rules: These criteria stress the importance of 5' terminal nucleotides for determining siRNA efficiency -sense 5' GC-rich, antisense 5' AU-rich and antisense positions 1-7 AU-rich. The rules are as follows:

- $\mathrm{G} / \mathrm{C}$ at position 1 (sense strand)

- $\mathrm{A} / \mathrm{U}$ at position 19 (sense strand)

- At least $5 \mathrm{~A} / \mathrm{U}$ in the 3 ' terminal (sense strand)

- No GC stretch longer than 9nt

\section{(e) Hsieh Rules:}

- No C at position 6 (sense strand)

- $\mathrm{C} / \mathrm{G}$ at position 11 (sense strand)

- A at position 13 (sense strand)

- $\mathrm{G}$ at position 16 (sense strand)

- U at position 19 (sense strand)

- No $\mathrm{G}$ at position 19 (sense strand)

(f) Reynolds rules: Reynolds et al. recently proposed a set of additional rules that improves the efficiency of RNAi significantly when applied together. Each rule is assigned a score, which is summed up to a total duplex score. Out of a set with scores greater than 6 , only about $17 \%$ were non-functional in their study, but as is shown in results on independent test sets were not so successful, which can be caused by over fitting to the training data.

\section{Other rules}

(a) Khvorova rule: The results suggest that the thermodynamic properties play a critical role in duplex unwinding and strand retention by RISC. The rules are as follows:

- Low stability 3' (sense strand) $>-8.5[\mathrm{kcal} / \mathrm{mol}]$

- Low stability 6-11 (sense strand) $>-7[\mathrm{kcal} / \mathrm{mol}]$

- High stability 5' (sense strand) $<-9[\mathrm{kcal} / \mathrm{mol}]$

(b) Takasaki rules: These rules suggested that an ideal regular expression is "GN $\{4\}$ AGAUN $\{5\} \mathrm{UN}\{3\}[\mathrm{G}] \mathrm{N} *$ ". The rules are

- No A/U at position 1 (sense strand)

- $\mathrm{G}$ at position 1 (sense strand)

- A at position 6 (sense strand)

- $\mathrm{G}$ at position 7 (sense strand)

- No U at position 7 (sense strand)

- A at position 8 (sense strand)

- No G at position 8 (sense strand)

- No G at position 9 (sense strand)

- U at position 9 (sense strand)

- U at position 15 (sense strand)

- No $\mathrm{G}$ at position 19 (sense strand)

Figure 1

(c) Schwarz rules: The experimental result of this study is that 
Citation: Gupta SK, Akhoon BA, Srivastava M, Gupta SK (2010) A Novel Algorithm to Design an Efficient siRNA by Combining the Pre Proposed Rules of siRNA Designing. J Comput Sci Syst Biol 3: 005-009. doi:10.4172/jcsb.1000048

3' end sense strand must be less energetic then 5' end. The rules are

(d) Chalk (stockholm rules):

- Total hairpin energy $<1[-\mathrm{kcal} / \mathrm{mol}]$

- Antisense 5' end binding energy < 9 [-kcal/mol]

- Sense 5' end binding energy in range 5-9 exclusive [-kcal/ $\mathrm{mol}]$

- GC content between $36 \%$ and $53 \%$

- Middle (7-12) binding energy < 13 [-kcal/mol]

- 5' sense strand -5' antisense strand Binding Energy Differ ence $<0[-\mathrm{kcal} / \mathrm{mol}]$

- Energy Difference within -1 and 0 [-kcal/mol]

\section{Strategy}

Source code of algorithm was used to find out all the possible target sites distributed among retrieved Fasta sequences. All the siRNA were designed on the basis of target sites. Blast algorithm was applied for homology search and that of Fasta algorithm to detect off-target sequences. Compared the designed siRNA with target mRNA. Finally, the effectiveness of siRNA, secondary structure, duplex temperature, free energy, and specific effect (interaction with other biochemical pathways) of designed siRNA were calculated.

\section{Results}

siRNA predicted by ambion siRNA target finder

Target sequence 1: AATGCGGGGTTAATTTAACTC

Sense strand siRNA: UGCGGGGUUAAUUUAACUCtt Antisense strand siRNA: GAGUUAAAUUAACCCCGCAtt

Target sequence 2: AATTTAACTCAGCCTCTGTGT

Sense strand siRNA: UUUAACUCAGCCUCUGUGUtt Antisense strand siRNA: ACACAGAGGCUGAGUUAAAtt

Target sequence 3: AACTCAGCCTCTGTGTGAGTG

Sense strand siRNA: CUCAGCCUCUGUGUGAGUGtt Antisense strand siRNA: CACUCACACAGAGGCUGAGtt

Target sequence 4: AACCCTCAGCTTAGCATGGGA

Sense strand siRNA: CCCUCAGCUUAGCAUGGGAtt Antisense strand siRNA: UCCCAUGCUAAGCUGAGGGtt

Target sequence 5: AAGTAGCTTCCCTGTTGACCC Sense strand siRNA: GUAGCUUCCCUGUUGACCCtt Antisense strand siRNA: GGGUCAACAGGGAAGCUACtt

siRNA predicted by siRNA program of mEmboss $>11 \_248 \%$ GC 50.0 Score 10

gcatgtgtctgctgctccctagtctgggccatgagtgagggtggaggccaa gtctcatgcaactttccattgaaggcccttt

$>11 \_63 \%$ GC 55.0 Score 8

gcatgtgtctgctgctcctagtctgggccatgagtgagggtggaggccaagtctcat gcaactcagcctctgtgtgagtgga

$>11 \_108 \%$ GC 55.0 Score 8

gcatgtgtctgctgctcctagtctgggccatgagtgagggtggaggccaagtctcat gcaacctcagcttagcatgggaag

$>11 \_128 \%$ GC 55.0 Score 8

gcatgtgtctgctgctccetagtctgggccatgagtgagggtggaggccaagtctcatgcaa gtagcttccetgttgaccetg

\section{$>1158 \%$ GC 40.0 Score 7}

gcatgtgtctgctgctccetagtctgggccatgagtgagggtggaggccaagtctcatgcaattt aactcagcctctgtgtga

>11_114\%GC 50.0 Score 7 gcatgtgtctgctgctccctagtctgggccatgagtgagggtggaggccaagtctcatgcca gcttagcatgggaagtagctt

\section{$>11 \_173 \%$ GC 60.0 Score 7}

gcatgtgtctgctgctccctagtctgggccatgagtgagggtggaggccaagtctcatgcaa ggtgtgggcaccatttggecc

\section{$>11 \_243 \%$ GC 40.0 Score 7}

gcatgtgtctgctgctcctagtctgggccatgagtgagggtggaggccaagtctcatgcaa gacaactttccattgaaggcc

$>11 \_247 \%$ GC 50.0 Score 7

gcatgtgtctgctgctcctagtctgggccatgagtgagggtggaggccaagtctcatgcc aactttccattgaaggecctt

\section{$>11 \_62 \%$ GC 50.0 Score 6}

gcatgtgtctgctgctccetagtctgggccatgagtgagggtggaggccaagtctcat gctaactcagcctctgtgtgagtgg

\section{$>11 \_79 \%$ GC 50.0 Score 6}

gcatgtgtctgctgctcctagtctgggccatgagtgagggtggaggccaagtctcatgc gagtggatgattcaggttgccag

\section{$>11 \_87 \%$ GC 50.0 Score 6}

Gcatgtgtctgctgctccctagtctgggccatgagtgagggtggaggccaagtctcatgc gattcaggttgccagagacagaa

\section{siRNA predicted by java tool based on designed algorithm}

\begin{tabular}{|c|c|c|}
\hline Rank & Position & isense sequence \\
\hline 1 & 160 & AAGCUGAGGGUUCUGUCUCUG \\
\hline 2 & 45 & AAAUGCAUGAGACUUGGCCUC \\
\hline 3 & 109 & UGAGUUAAAUUAACCCCGCAU \\
\hline 4 & 294 & AAAGUU GUCUUCCCUGCUCAG \\
\hline 5 & 32 & UUGGCCUCCACCCUCACUCAU \\
\hline 6 & 290 & UUGUCUUCCCUGCUCAGCCCC \\
\hline 7 & 105 & UUAAAUUAA CCCCGCAUUCAA \\
\hline 8 & 299 & AAUGGAAAGUUGUCUUCCCUG \\
\hline 9 & 99 & UAACCCCGCAUUCAAUGAGGG \\
\hline 10 & 231 & AAAUGGUGCCCACACCUUCCA \\
\hline 11 & 396 & ACACUUGGCAUGAGGUCGCCU \\
\hline 12 & 264 & UGCUGCUCUCUUCAGAGCUGU \\
\hline 13 & 268 & UUCCUGCUGCUCUCUUCAGAG \\
\hline 14 & 36 & AGACUUGGCCUCCACCCUCAC \\
\hline 15 & 302 & UUCAAUGGAAAGUUGUCUUCC \\
\hline 16 & 230 & AAUGGUGCCCACACCUUCCAA \\
\hline 17 & 240 & AACUGGGCCAAAUGGUGCCCA \\
\hline 18 & 156 & UGAGGGUUCUGUCUCUGGCAA \\
\hline 19 & 46 & AAAAUGCAUGAGA CUUGGCCU \\
\hline 20 & 173 & UACUUCCCAUGCUAAGCUGAG \\
\hline 21 & 254 & UUCAGAGCUGUAAGAACUGGG \\
\hline 22 & 143 & UCUGGCAACCUGAAUCAUCCA \\
\hline 23 & 220 & ACACCUUCCAAGCCAACCUCA \\
\hline 24 & 104 & UAAAUUAACCCCGCAUUCAAU \\
\hline 25 & 374 & ACUCUCACCCCUCAUGGGCAG \\
\hline
\end{tabular}

$\begin{array}{cc}\text { (+)ve Value } & (-) \text { ve value } \\ 248 & -31 \\ 268 & -6 \\ 265 & -7 \\ 278 & 22 \\ 270 & 23 \\ 232 & -3 \\ 273 & 40 \\ 226 & 9 \\ 267 & 53 \\ 241 & 30 \\ 266 & 76 \\ 147 & -38 \\ 212 & 29 \\ 235 & 53 \\ 190 & 13 \\ 170 & -5 \\ 168 & 3 \\ 227 & 69 \\ 181 & 24 \\ 175 & 18 \\ 143 & -13 \\ 204 & 49 \\ 250 & 98 \\ 247 & 96 \\ 232 & 82 \\ & \end{array}$

\section{Comparative efficacy}

Table 2 listed comparative results of duplex energies and melting temperatures predicted by proposed algorithm based tool and ambion and mEmboss software.

\begin{tabular}{|c|c|c|c|}
\hline & Overall Energy & Duplex Energy & $\begin{array}{c}\text { Melting } \\
\text { Temperature }\end{array}$ \\
\hline Ambion & -26.3 & -26.7 & 79.8 \\
\hline mEmboss & -28.3 & -29.7 & 81 \\
\hline Our Tool & -30.1 & -33.0 & 86.3 \\
\hline
\end{tabular}

Table 2: Duplex energies and Melting temperatures of predicted siRNA.

\section{Discussion}

The melting temperature were calculated from the duplex formed by antisense siRNA and the target mRNA having more in siRNAs which are design by our siRNA tool. This result indicates that more accurate and greater tendency of silencing is shown by siRNA designed by our siRNA designing tool. The value of duplex energy and overall energy were comparatively 


\section{Journal of Computer Science \& Systems Biology - Open Access JCSB/Vol.3 Issue 1}

less which indicates that the design siRNA by our tool will be more stable.

Our siRNA designing tool was built on for every platform because java/j2ee platform were used which is platform independent. The databases involved were implemented locally to speed up the design process using a MYSQL database. MySQL is used as the relational database management system to host a local database. JSP, SERVLET and HTML scripts were written to communicate with the databases using a web based user interface (GUI interface) and java programming language was used to implement the design algorithms as well as to parse BLAST and FASTA output. MATLAB statistical software was used to analyze the siRNA data. Tool uses a variety of information from genetic databases such as NCBI RefSeq and Human UniGene database, similarity searching software such as BLAST and FASTA to confirm the uniqueness of siRNA design. Web tool users need not install the siRNA design tool locally; BLAST searches carried out in the program were performed using the program BLASTN from the NCBI standalone BLAST package with standard settings and no filtering. The web-based front-end of the program, as well as the in silico digestion of the input query, quality control algorithm and output parsing scripts, were written in the java programming language. The JSP/servlet for interactive selection of sequence and manipulation of penalty scores from the graphical output was programmed in Java. The software accepts input of one or multiple target genes in Genbank or FASTA formats. Since the Genbank format provides locations for the coding region of the gene (CDS), it is the preferred format used in this study. Once the start location is determined for each gene sequence, the selection process starts by collecting siRNA candidates. It shifts one nucleotide each time along the sequence to exhaust all potential siRNA sequences and avoids any sequences that contain uncertain nucleotides other than $\mathrm{A}$, $\mathrm{T} / \mathrm{U}, \mathrm{G}$, or $\mathrm{C}$ because these regions may have single nucleotide polymorphism, or SNP. The selection process is the major advantages of this tool are that it allows users to adjust all the selection criteria or even rearrange the filters in the three phases through a configuration file. Where users can adjust the following from the graphic user interface (GUI) of this tool: the length of the siRNA, the range of GC content and the definition of polymers of $A, U / T, G$ and $C$, etc.

\section{Conclusion}

Most of designed siRNA shows off-target effect, which can be reduced by Smith Waterman searching but complete off-targeting can be removing by chemical modification of nucleotide bases. The stability of siRNAs can be increase by convert it into short hairpin loop form. Algorithm calculated the RNA secondary structure and minimum free energy for each target sense and anti-sense sequences. Sequences were filtered to remove candidates with unfavored thermodynamic property. Effective siRNAs have a relatively lower duplex stability(Tm; less stable, more A/ U rich) toward the 5'-end of the strand that remains in RISC (the 'guide strand') and a relatively higher Tm (more stable, more G/ C rich) toward the 5'-end of the degraded or ejected strand.

\section{References}

1. Bernstein E, Caudy AA, Hammond SM, Hannon GJ (2001) Role for a bidentate ribonuclease in the initiation step of RNA interference. Nature 409: 363-366. »CrossRef » PubMed » Google Scholar

2. Cogoni C, Macino G (2000) Post-transcriptional gene silencing across kingdoms. Curr Opin Genet Dev 10: 638-643. »CrossRef » PubMed » Google Scholar

3. Denli AM, Hannon GJ (2003) RNAi: An ever-growing puzzle. Trends Bioche Sci 28: 196-201. »CrossRef » PubMed » Google Scholar

4. Fire A, Xu S, Montgomery MK, Kostas SA, Driver SE, et al. (1998) Potent and specific genetic interference by double-stranded RNA in Caenorhabditis elegans. Nature 391: 806-811. » CrossRef » PubMed » Google Scholar

5. Grishok A, Pasquinelli AE, Conte D, Li N, Parrish S, et al. (2001) Genes and mechanisms related to RNA interference regulate expression of the small temporal RNAs that control C. elegans developmental timing. Cell 106: 23-34. »CrossRef » PubMed » Google Scholar

6. Hannon GJ (2002) RNA interference. Nature 418: 244-251. »CrossRef » PubMed " Google Scholar

7. Hammond SM, Boettcher S, Caudy AA, Kobayashi R, Hannon GJ (2001) Argonaute2, a link between genetic and biochemical analyses of RNAi. Science 293: 1146-1150. » CrossRef » PubMed » Google Scholar

8. Hong Z, Xiao Z, Yufang W, Benjamin RS (2006) A Three-Phase Algorithm for Computer Aided siRNA Design. Informatica 30: 357-364. »CrossRef » PubMed » Google Scholar

9. Hutvagner G, McLachlan J, Pasquinelli AE, Balint E, Tuschl T, et al. (2001) A cellular function for the RNA-interference enzyme Dicer in the maturation of the let-7 small temporal RNA. Science 293: 834-838. »CrossRef » PubMed » Google Scholar

10. Hutvagner G, Zamore PD (2002a) RNAi: Nature abhors a double-strand. Curr Opin Genet Dev 12: 225-232. »CrossRef » PubMed » Google Scholar

11. Hutvagner G, Zamore PD (2002b) A microRNA in a multiple-turnover RNAi enzyme complex. Science 297: 2056-2060. »CrossRef » PubMed » Google Scholar

12. Jayasena SD (2006) Designer siRNAs to overcome the challenges from the RNAi pathway. J RNAi Gene Silencing 2: 109-117. »CrossRef » PubMed » Google Scholar

13. Ketting RF, Fischer SE, Bernstein E, Sijen T, Hannon GJ, et al. (2001) Dicer functions in RNA interference and in synthesis of small RNA involved in developmental timing in C. elegans. Genes Dev 15: 2654-2659. »CrossRef » PubMed » Google Scholar

14. Knight SW, Bass BL (2001) A role for the RNase III enzyme DCR-1 in RNA interference and germ line development in Caenorhabditis elegans. Science 293: 2269-2271. »CrossRef » PubMed » Google Scholar

15. Nykanen A, Haley B, Zamore PD (2001) ATP requirements and small interfering RNA structure in the RNA interference pathway. Cell 107: 309-321. »CrossRef » PubMed » Google Scholar

16. Martinez J, Patkaniowska A, Urlaub H, Luhrmann R, Tuschl T (2002) Singlestranded antisense siRNAs guide target RNA cleavage in RNAi. Cell 110: 563-574. » CrossRef » PubMed » Google Scholar 